\title{
Noncontiguous protein interaction domains in osteopontin contribute to enhance HIV-I replication
}

\begin{abstract}
Osteopontin (OPN) is a proinflammatory cytokine produced by T-cells, macrophages, osteoclasts, and several other cell types, which confers immunity to many intracellular pathogens. OPN was first identified as an early marker of cellular activation of T-lymphocytes and subsequently was shown to play a role in cancer through its ability to promote cell survival and inflammation. OPN levels are elevated in the plasma and cerebrospinal fluid of HIV-infected individuals and even more so in those suffering from HIV-related neurocognitive impairment. The infiltration of monocytes and macrophages both infected and uninfected into the brain is the first step in HIV pathogenesis of the central nervous system. Inhibition of OPN in macrophages significantly impairs HIV replication. In an effort to identify and understand the role of OPN in the neuropathogenesis of HIV infection, we are using a combination of in vitro, ex vivo and in vivo approaches. In this study we have used a molecular approach and a surrogate cell culture model to identify the domains of OPN that are required to enhance HIV replication. We found that N- and C-terminal fragments, encoding multiple motifs including sequences involved in binding integrins and CD44, a domain know to promote adhesion contribute to OPN's ability to increase HIV replication. Use of inhibitors against c-Jun N-terminal kinase (JNK) and phosphoinositide 3-kinase (PI-3K) impaired the ability of OPN to increase the integrin subunit 1 or CD29 on the surface of HIV-infected and bystander cells. These results suggest that multiple OPN-regulated cellular pathways are commandeered by HIV to promote productive replication and cell-to-cell spread.
\end{abstract}

Keywords: Syncytia, Beta-1 integrin, CD44, CD29, Phosphoinositide-3 kinase, JNK, Secreted phosphoprotein-1
Volume I Issue I - 2014

\author{
Caitlin Eger,' Kimberly Cirelli, ${ }^{2}$ Jessica \\ Budiaman, ${ }^{3}$ Amanda Brown ${ }^{4}$ \\ 'Department of Neurology, Syracuse University, USA \\ ${ }^{2}$ Department of Neurology, Massachusetts Institute of \\ Technology, USA \\ ${ }^{3}$ Department of Neurology, School of Public Health, Tufts \\ University, USA \\ ${ }^{4}$ Department of Neurology, Johns Hopkins University School of \\ Medicine, USA
}

Correspondence: Amanda Brown, Department of Neurology, Johns Hopkins University School of Medicine, Baltimore, MD, USA, Tel 4I0-6I4-2429; Fax 4I0-502-6737,

Email abrown76@jhmi.edu

Received: May 05, 2014 | Published: May 23, 2014

\section{Introduction}

Viruses such as HIV-1 have evolved to coexist with their hosts by usurping cellular pathways and signaling mechanisms that indirectly promote viral spread while evading the immune response. In a screen to identify host cell factors that are up regulated in macrophages infected with HIV, we found that the proinflammatory cytokine osteopontin (OPN) was significantly elevated. ${ }^{1}$ An increase of OPN RNA using microarray analyses was found in the brains of encephalitic SIVinfected rhesus macaques. ${ }^{2}$ Additional studies revealed that OPN is significantly elevated in the plasma and cerebrospinal fluid of HIVinfected individuals with cognitive impairment. ${ }^{1,3} \mathrm{OPN}$ is chemotactic for monocytes and may exacerbate HIV central nervous system disorder by promoting the recruitment of these cells to the brain, as well as promoting their survival by stimulating anti-apoptotic pathways. ${ }^{4,5}$ We found that siRNA-mediated knockdown of OPN in primary human macrophages reduced HIV replication fifty percent, highlighting a direct role for OPN on the viral life cycle. ${ }^{1}$ Using a heterologous cell culture model, which is susceptible to HIV-1, we showed that ectopic expression of OPN increases the degradation of the I $\kappa \mathrm{B}$ inhibitor allowing NF- $\mathrm{KB}$ to translocation to the nucleus to drive HIV-LTR promoter activity. Indeed, deletion of the NF- $\kappa B$ binding sites in the HIV-LTR abrogated the effect of OPN. ${ }^{1}$

First described as an early activation marker for T-cells (ETA1), the gene for OPN, SPPI(secreted phosphoprotein-1) encodes a multifunctional protein that is elevated in many cancers, autoimmune and inflammatory disorders. ${ }^{6} \mathrm{OPN}$ is a target for post-translational modifications including glycosylation, phosphorylation and cleavage by thrombin and metalloproteinase. ${ }^{5,7}$ When cleaved at its thrombin target site, the $\mathrm{N}$ - and C-terminal halves of OPN were shown to be functional, regulating the balance between IL-10 and IL-12 production and influencing cell-mediated immunity. ${ }^{8}$ Three isoforms for OPN, OPNa, OPNb and OPNc have been identified and several studies suggest that they may be expressed in a tissue and or disease specific manner. ${ }^{9}$ OPN can be secreted from cells after processing of its signal peptide. In addition, the presence of an alternative start codon allows for an intracellular variant of OPN to be made which has been shown to function in the recruitment of dendritic cells. ${ }^{10}$ OPN has several discrete motifs including an aspartate domain followed by recognition sites for integrin binding RGD and SVVYGLR in the $\mathrm{N}$-terminal portion, as well as putative calcium-, heparin-, and CD44binding regions in the $\mathrm{C}$-terminal half of the protein. ${ }^{5,7}$

In order to better understand the molecular mechanisms by which OPN enhances HIV replication we performed deletion analyses and infectivity studies using the $\mathrm{OPNb}$ isoform, which we found to be up regulated in HIV-infected macrophages, ${ }^{1}$ to identify the minimal domain of OPN that can function in this regard. Given the precedence in the literature regarding the modular nature of OPN and the fact that cleaved forms of the protein are functional, suggested that a deletion analyses study would be informative. Unexpectedly, we found that multiple non-contiguous regions of OPN could enhance HIV replication just as well as the full-length protein. In this regard, signaling through the aspartate and integrin binding domains appears to contribute toward HIV promoter activity and the upregulation of 
$\beta 1$ integrin on infected and bystander or uninfected cells in the same culture, while the calcium- and heparin-, CD44-binding domains enhance cell-to-cell fusion and viral spread. Moreover, as the $\mathrm{N}$-terminal truncations lacked signal sequences, our data suggest that OPN can function through intracellular signaling pathways to enhance HIV spread. Indeed, inhibitor studies implicated phosphoinositide-3 kinase (PI-3K) and c-Jun N-terminal kinase (JNK) pathways in these functions. Together, these data suggest that HIV life cycle intersects with and benefits from several innate OPN signaling pathways.

\section{Materials and methods}

\section{Cloning of SPP I deletion mutants}

The plasmid encoding the full length SPP1 cDNA was obtained from OriGene (\#TC127388) and used as a template with primers to generate DNA fragments encoding deletions of the gene (Table 1). The amplified sequences were cloned into pCMV6-XL4 using the $\mathrm{SacI} / \mathrm{XhoI}$ sites. Restriction enzyme analyses and DNA sequencing were used to confirm sequence integrity.

\section{HIV infectivity assay}

TZM-bl cells were obtained from the NIH AIDS Research and Reference Reagent Program (\#080122) from J. Kappes and X. Wu Table I Primer sequences and Tranzyme Inc. and cultured in DMEM medium containing 10\% FBS with $1 \%$ penicillin, streptomycin and sodium pyruvate. TZM-bl cells, which express the CD4 and CCR 5 co receptor for susceptibility to HIV infection were placed on 24-well plates (Costar Cell Bind) and the next day transfected with plasmids expressing full-length or SPP1 deletion constructs using lipofectamine in Opti-MEM medium. After $\sim 18$ hrs later the transfected cells were allowed to recover in DMEM complete media for $3 \mathrm{hrs}$ prior to infection with $\mathrm{HIV}_{\mathrm{SF} 162} \mathrm{R} 3-\mathrm{GFP}^{11}$ overnight in DMEM with $2 \%$ FBS. After $72 \mathrm{hrs}$ post-infection supernatants were harvested for flow cytometry and analyzed for GFP and or $\beta 1$ integrin expression using anti-CD29-PE antibody (Clone MEM-101A Invitrogen) on the FACS Calibur(BD Biosciences).

\section{Microscopy and fusogenicity analyses}

Images of monolayers were captured after 2-3 days after infection on a Zeiss AxioObserver A1 inverted microscope using a 20x objective and processed using Adobe Photoshop to adjust the brightness and contrast and sharpen with the filter, unsharp mask in the same manner for each image. The entire area of the 24-well plate was inspected and five images captured to compare the number and area covered by the fused cells for each $S P P 1$ deletion compared to the SPP1 wild type control.

\begin{tabular}{|c|c|c|}
\hline Primer* & Sequence & Notes \\
\hline SPP-N-F & GGGATCCGCCACAACCATGAGAATTGCAGTGATTTGCTTTTGCCT & $\begin{array}{l}\text { Kozak sequence } \\
\text { present }\end{array}$ \\
\hline SPP-Asp-F & GGGATCCGCCACAACCATGAGAATTGCAGTGATTTGCTTTTGCCT & Same as SPP-N-F \\
\hline SPP-Asp-R & GCCTCGAGTTATTTATCGTCGTCATCCTTATAATCATCATATGTGTCTACTGTGGGGACA & $\begin{array}{l}\text { Without RGD } \\
\text { sequence }\end{array}$ \\
\hline SPP-SVV-F & GGGATCCGCCACAACCATGAGAATTGCAGTGATTTGCTTTTGCCT & Same as SPP-N-F \\
\hline SPP-SVV-R & GCCTCGAGTTATTTATCGTCGTCATCCTTATAATCCAGTCCATAAACCACACTATCACCT & \\
\hline SPP-Thr6-F & GGGATCCGCCACAACCATGAGAATTGCAGTGATTTGCTTTTGCCT & Same as SPP-N-F \\
\hline SPP-Thr6-R & GCCTCGAGTTATTTATCGTCGTCATCCTTATAATCTTTTGACCTCAGTCCATAAACCACAC & \\
\hline SPP-Tail7-F & GGGATCCGCCACAACCATGAGAATTGCAGTGATTTGCTTTTGCCT & Same as SPP-N-F \\
\hline SPP-Tail7-R & GCCTCGAGTTATTTATCGTCGTCATCCTTATAATCAGAAGGCGCGTTCAGGTCCT & \\
\hline SPP-Ca+8-F & GGGATCCGCCACAACCATGAGAATTGCAGTGATTTGCTTTTGCCT & Same as SPP-N-F \\
\hline SPP-Ca+8-R & GCCTCGAGTTATTTATCGTCGTCATCCTTATAATCCGTTTCATAACTGTCCTTCCCACG & \\
\hline SPP-Nr2-F & CACCGGAGCTCGCCACAACCATGGAACGACTCTGATGATGTAGATGACAC & \\
\hline SPP-Nr2-R & GCCTCGAGTTATTTATCGTCGTCATCCTTATAATCATTGACCTCAGAAGA & \\
\hline SPP-Aspr3-F & CACCGGAGCTCGCCACAACCATGGACTCTGATGATGTAGATGACACTGAT & \\
\hline SPP-Aspr3-R & GCCTCGAGTTATTTATCGTCGTCATCCTTATAATCATTGACCTCAGAAGA & Same as SPP-Nr2-R \\
\hline SPP-RGD-F & CACCGGAGCTCGCCACAACCATGGGCCGAGGTGATAGTGTGGTTTAT & \\
\hline SPP-RGD-R & GCCTCGAGTTATTTATCGTCGTCATCCTTATAATCATTGACCTCAGAAGA & Same as SPP-Nr2-R \\
\hline SPP-SVVr-F & CACCGGAGCTCGCCACAACCATGAGTGTGGTTTATGGACTGAGGTCAAAA & \\
\hline SPP-SVVr-R & GCCTCGAGTTATTTATCGTCGTCATCCTTATAATCATTGACCTCAGAAGA & Same as SPP-Nr2-R \\
\hline SPP-Thr $\Delta-\mathrm{F}$ & GGAGCTCGCCACAACCATGAAATCTAAGAAGTTTCGCAGACCTGACA & \\
\hline SPP-Thr $\Delta-\mathrm{R}$ & GCCTCGAGTTATTTATCGTCGTCATCCTTATAATCATTGACCTCAGAAGA & Same as SPP-Nr2-R \\
\hline SPP-Ca2-F & GGAGCTCGCCACAACCATGGATTGGGACAGCCGTGGGAA & \\
\hline SPP-Ca2-R & GCCTCGAGTTATTTATCGTCGTCATCCTTATAATCATTGACCTCAGAAGA & Same as SPP-Nr2-R \\
\hline
\end{tabular}

*Bam $\mathrm{HI}$ restriction site at $\mathrm{N}$-termini and $\mathrm{Xho} \mathrm{I}$ at $\mathrm{C}$-terminus with FLAG epitope and stop codon. 


\section{Treatment with kinase inhibitors}

TZM-bl cells were transfected and infected with HIV as described above and on the next day culture medium containing one of the following inhibitors was added: U73122 $(1 \mu \mathrm{M})$, PD98059 $(10 \mu \mathrm{M})$, SB203580 $(10 \mu \mathrm{M})$, SP600125 $(10 \mu \mathrm{M})$ (Sigma-Aldrich) and LY294002 $(50 \mu \mathrm{M})$ (Invitrogen) were dissolved in DMSO. The inhibitors at the indicated concentrations did not have any impact on cell viability during the assay period. The cells were harvested 3-4 days after infection and quantified for infectivity and $\beta 1$ integrin expression by flow cytometry.

\section{Results and discussion}

\section{Motifs in the $\mathrm{C}$ - and $\mathrm{N}$-termini of osteopontin (OPN) are sufficient to enhance HIV replication}

OPN is subject to several post-translational modifications such as phosphorylation, glycosylation and to cleavage by thrombin. OPN has a signal peptide and can be secreted however intracellular forms of the protein have also been reported..$^{10}$ Interestingly, early studies showed that cleavage of OPN at the thrombin site results in two proteins that were functionally active..$^{5,8}$ Hence, these data strongly suggested that OPN possesses protein domains that can function independently. Therefore, to determine the minimal domain of OPN that could enhance HIV-1 replication, a series of FLAG-epitopetagged SPP1 (gene for OPN) deletion mutants were generated by PCR and cloned into the mammalian expression vector pCMV6-XL4 All of the C-terminal truncations encoded a signal sequence at the $\mathrm{N}$-terminus, while the amino-terminal deletions did not (Figure 1). TZM-bl cells which express the CD4 and CCR5 co-receptor thus making them susceptible to HIV infection were transfected with each SPP1 deletion mutant or with plasmids encoding the full length gene and $18 \mathrm{hrs}$ later, infected with $\mathrm{HIV}_{\mathrm{SF} 162} \mathrm{R} 3$-GFP to determine whether the mutants retained the ability to enhance HIV replication. Interestingly, no consistent significant differences in the ability to enhance HIV infectivity between SPP wt (wild type) and the deletion mutants, as reflected by the number of GFP+ cells was observed over several independent experiments. However, for the SPP-SVVr mutant, a significant decrease in its ability to increase HIV-1 promoter activity as revealed by the mean fluorescent intensity (MFI) of GFP compared to wild type, SPP1-Asp1, SPP1-Thr6, SPP1-Ca+8, SPP1$\mathrm{Nr}$ 2, SPP1-RGD, and SPP1-Ca was seen (GFP MFI) (Figure 2a). Interestingly, addition of four amino acid residues involved in integrin binding, GRGD, to the N-terminus of SPP1-SVVr was sufficient to restore activity to wild type levels (Figure 2a). The enhancement phenotype of the C-terminal SPP1- Thrdel fragment trended toward less activity than wild type, but was variable, while the SPPI-Ca fragment had wild type activity (Figure 2a). Collectively, these results suggest that the N-terminal aspartate and integrin binding domains are sufficient to enhance HIV infectivity and replication, but that the putative calcium binding and heparin-binding domains also possess this activity independently. Hence, noncontiguous domains in OPN contribute to enhance HIV-1 replication in TZM-bl cells.

\section{OPN increases CD29 integrin levels on infected cells}

The OPN deletion analyses suggested that the integrin binding motifs played a critical role in enhancing HIV replication. To determine whether OPN-mediated enhancement of HIV replication involved alterations in the expression of its receptor integrin $\beta 1$ or CD29, we performed flow cytometric analyses to quantify the density of this receptor on HIV-infected GFP+ cells and on the bystander uninfected, GFP- cells in the same culture. Compared to cells not expressing SPP1, CD29 levels were significantly increased on HIV-infected cells transfected with the vector encoding SPP1 or the deletion mutants (Figure 2b), none vs all except Thrdel; $p=.0003$ to $<.0001, \mathrm{n}=9-12$ ). CD29 was significantly increased $\sim 3$-fold on HIV-infected cells expressing SPP1wt compared to bystander cells for $S P P 1 \mathrm{wt}$ (Figure 2b), (SPPwt-By) and all of the SPP1 deletion mutants examined (Figure 3), (Blue bracket, $\mathrm{p}<.0001$ ). No significant differences in the density of CD29 between SPP 1 wt and the SPPIdeletion mutants on HIV-infected cells were found. However, CD29 density on bystander cells expressing SPP1-Thrdel was significantly lower than on cells producing SPP1-Nr2, SPP1-Aspr3, SPP1-RGD, SPP1-SVVr, and $S P P 1-\mathrm{Ca}$ (Figure 2b). Interestingly, all of the former contain the C-terminal end of SPP1 and were progressively truncated from the amino-terminus (Figure 1). Collectively, these results suggest that HIV-1 replication alone induces the upregulation CD29expression and this increase is potentiated by OPN. Moreover, SPPIN terminal deletions, which have no signal peptide, retained significant ability to potentiate CD29 surface levels on HIV-infected cells suggesting that OPN can use intracellular signaling pathways to alter integrin receptor expression.

\section{OPN deletions enhance cell-to-cell fusion}

HIV-1 infection often results in the formation of cell-to-cell fusions called syncytium via the interactions between the viral envelope Gp120 and receptor/co-receptors CD4/CCR5 or CXCR4. Syncytium formation facilitates the spread of HIV from an initial locus to neighboring susceptible cells, thus greatly disseminating the virus. In carrying out the above studies we noticed that SPP1 transfected cells exhibited significantly enhanced HIV-induced syncytium formation compared to cells not expressing SPP1 (Figure 3) (control vs. OPN). The SPPIdeletion mutants varied from wild type in their ability to enhance syncytium formation. By comparing the size and quantifying the frequency of syncytia, a relative fusion induction score compared to wild type OPN was established (Table 2). Interestingly, N-terminal deletions SPP1-Thrdel and SPP1-Ca possessed near wild type ability to stimulate enhanced fusion (Figure 3, Table 2). Addition of the SVVYGLR motif to SPP1-Thrdel (SPP1-SVVr) greatly reduced its ability to enhance cell-to-cell fusion (Figure $3 \&$ Table 2). Likewise the addition of sequences upstream of the integrin motifs including the aspartate domain (SPP1-RGD, SPP1-Aspr3, SPP1-Nr2) did not enhance cell-to-cell fusion (Figure $3 \&$ Table 2). Truncation of the C-terminus (SPP1-Asp, $S P P 1-S V V)$ more strongly impaired fusogen formation compared to wild type and the N-terminal mutants SPP1$\mathrm{Thr} \triangle$ and SPP1-Ca (Figure 3). Interestingly, addition of sequences downstream of the integrin binding motifs increased the cell-to-cell fusion ability of SPP1-Thr6 and SPP1-Tail7 (Figure $3 \&$ Table 2). These data suggest that motifs in the C-terminal half of OPN are required for fusogenic activity and are involved in the facilitating HIV spread throughout the culture.

\section{Identification of signaling pathways involved in OPN mediated upregulation of $\beta I$ integrin subunit CD29 in HIV infection}

OPN-ligand CD44-receptor signaling has been linked to a phosphoinositide-3 (PI-3) kinase pathway, while OPN engagement of integrins can activate Ras/Src signaling leading to downstream stimulation of NF- $\mathrm{KB}$ function. ${ }^{5,7}$ To identify signaling pathways 
that contribute to OPN mediated upregulation of CD29 expression; we treated TZM-bl cells with kinase inhibitors targeting p38 MAPK, ERK, JNK, PI-3K, and phospholipase. Expression of SPP1 alone in the absence of any inhibitors significantly increased CD29 density on HIV-infected GFP+ and bystander cells (Figure 4, p<.0001). While inhibitors against p38 MAPK (SB203580), ERK (PD98059) and phospholipase (U73122) had no effect, treatment with SP600125(JNK) or LY294002 (PI-3K) significantly reduced the ability of SPP1 to potentiate CD29 expression on HIV-infected and bystander cells (Figure 4). These results suggest that signaling via JNK and PI-3K dependent pathways (s) are involved in OPN-mediated potentiating of CD29 expression in the context of HIV infection.

Figure I Schematic diagram of SPPI deletion mutants generated by PCR and cloned into a mammalian expression vector. Osteopontin is encoded by the SPPI gene and contains several motifs and domains: signal peptide (orange), sites for $\mathrm{N}$--linked glycosylation (pink), an aspartic acid rich domain (green), sequences involved in integrin binding (turquoise and blue), cleavage by thrombin (black) or metalloproteinases (brown), calcium binding (yellow) and heparin and CD44-- binding (white). The names of the truncation mutants are indicated beside each construct.

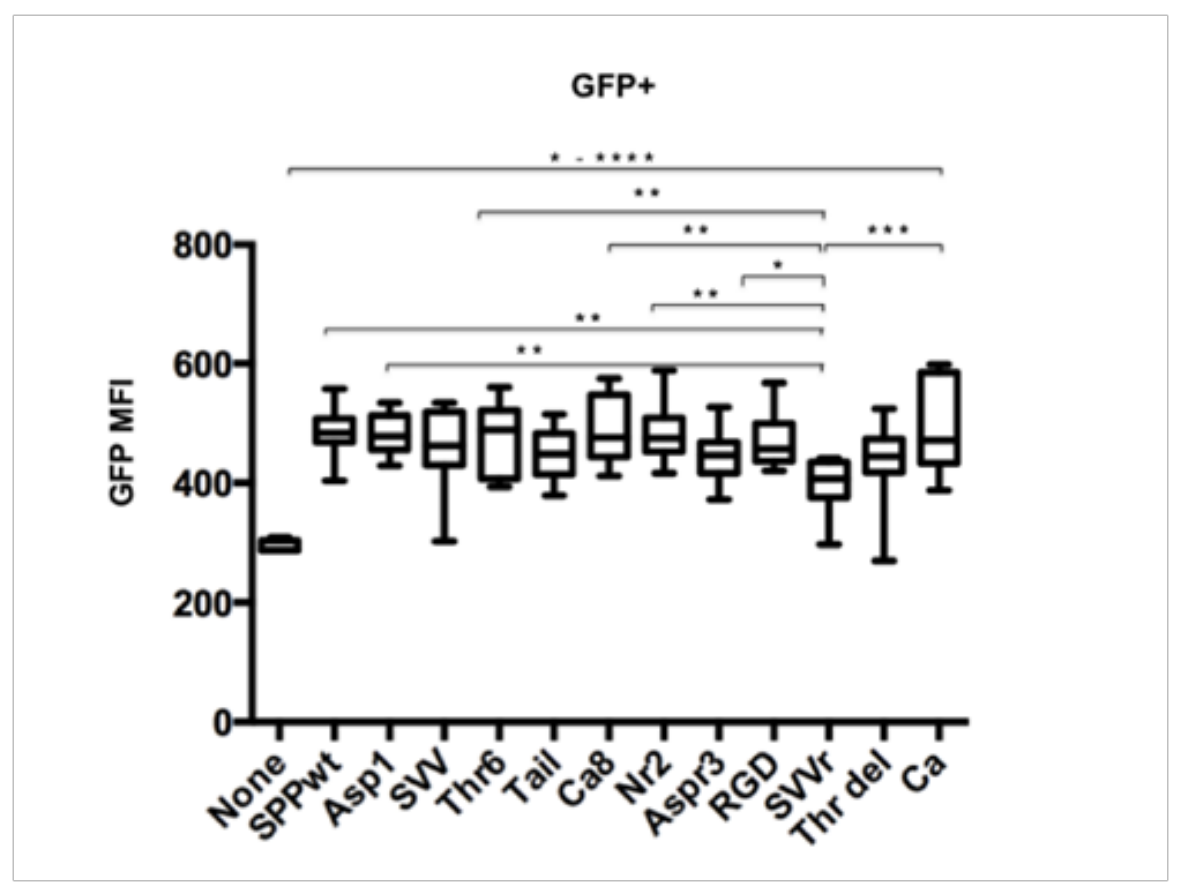

Figure 2a Reduction in HIV promoter activity with N--terminal truncation of SPPI.The mean fluorescent intensity (MFI) of GFP is an indirect measure of HIV--LTR promoter activity. One--way ANOVA with significance of $\mathrm{p}<.05$ was used. $* * * * \mathrm{p}<.000 \mathrm{I}, * * * \mathrm{p}<.0005, * * \mathrm{p}<.005,{ }^{*} \mathrm{p}<.05$. 


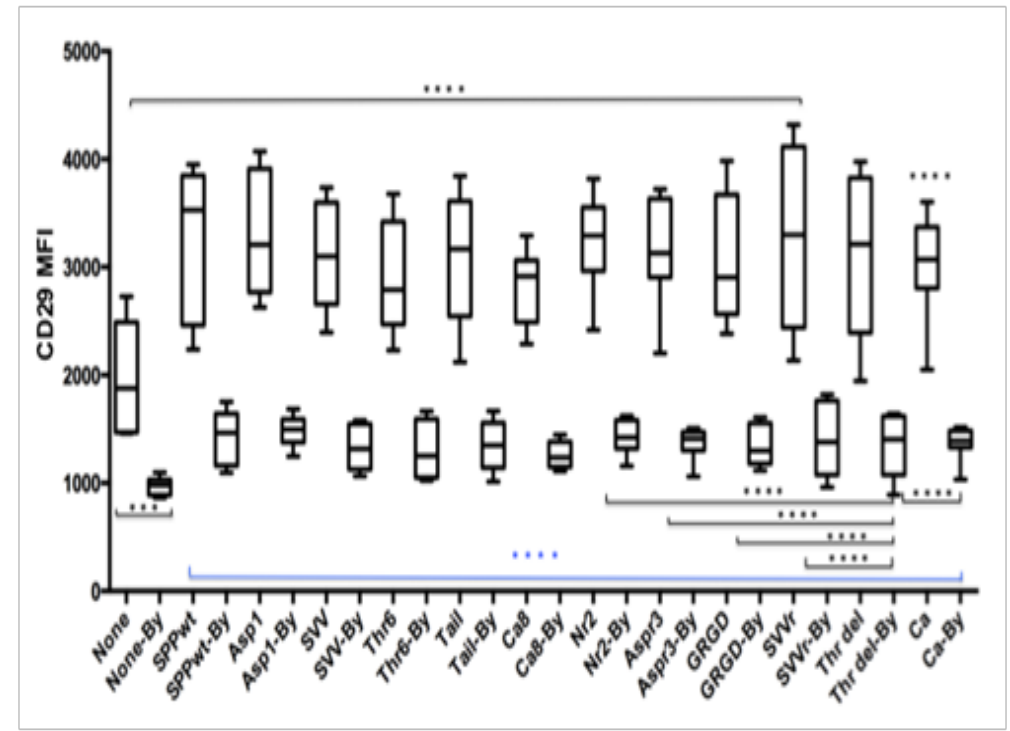

Figure 2b SPP/ wt and truncation mutants increase CD29 $\beta$ I integrin subunit on HIV--infected compared to bystander GFP negative cells. The mean fluorescent intensity (MFI) of CD29, a measure of the density of the receptor on the cell surface is shown for cells not expressing SPPI (None) and for each of the truncation mutants. One--way ANOVA with significance of $\mathrm{p}<.05$ was used. ${ }^{*} * * * p<.000 \mathrm{I}, * * * \mathrm{p}<.0005, * * p<.005,{ }^{*} \mathrm{p}<.05$.

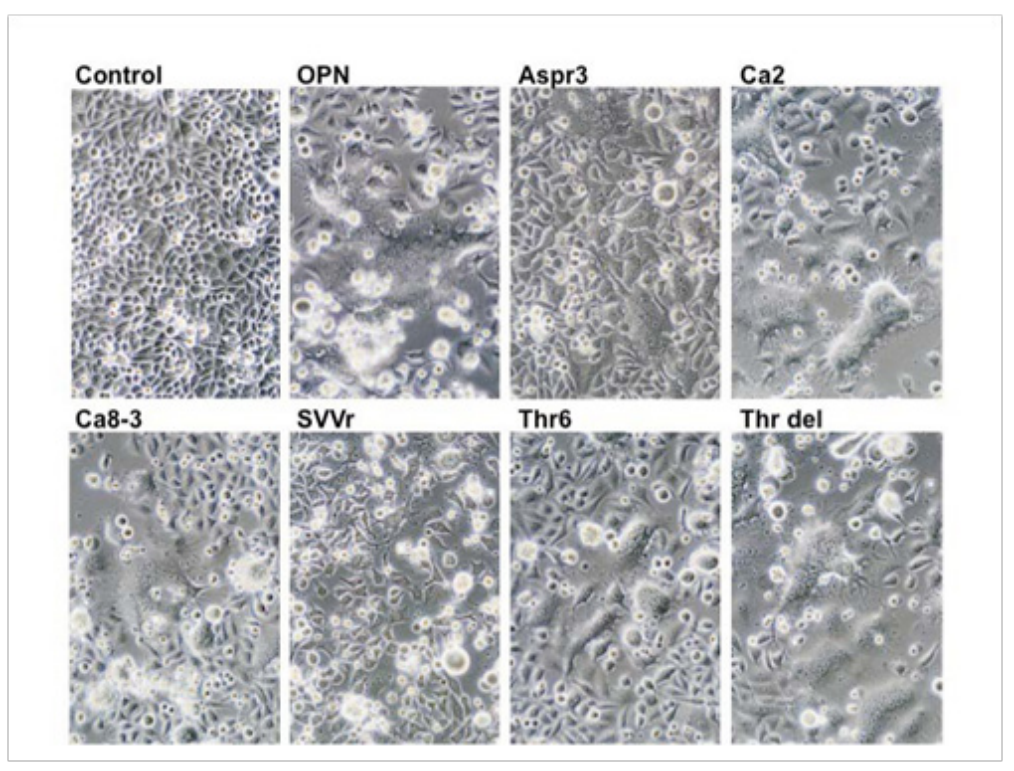

Figure 3 SPPI deletions enhance cell--to--cell fusion. Images of TZM--bl cells transfected with SPP/wt (OPN) or the indicated SPPI deletion mutants and infected with HIVSFI62--R3 GFP after 2--3 days post--infection are shown.

Table 2 Fusogenicity rank

\begin{tabular}{ll}
\hline Construct & Score \\
\hline SPP wild type & +++++ \\
SPP-Ca & ++++ \\
SPP-Thr & +++ \\
SPP-Tail7 & +++ \\
SPP-Thr6 & +++ \\
SPP-Ca+8 & ++ \\
SPP-RGD & ++ \\
SPP-Aspr3 & + \\
SPP-SVV & + \\
SPP-Nr2 & + \\
SPP-SVVr & + \\
SPP-AsP & $+/$ \\
\hline
\end{tabular}

Citation: Eger C, Cirelli K, Budiaman J, et al. Noncontiguous protein interaction domains in osteopontin contribute to enhance HIV-I replication. J Hum Virol Retrovirol. 2014; I(I):7-14. DOI: 10.15406/jhvrv.20I4.01.00003 


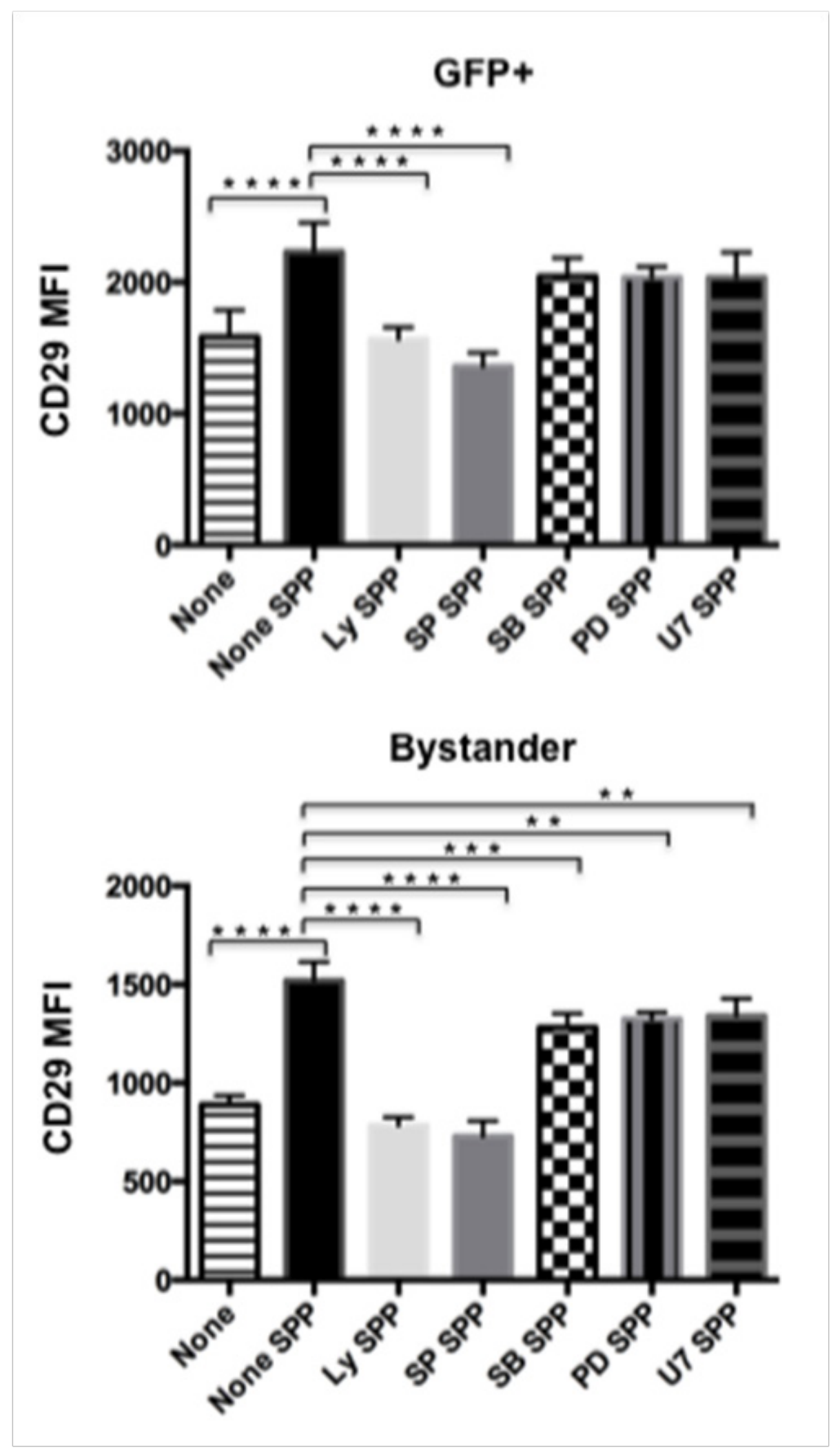

Figure 4 Inhibition of PI3K or JNK signaling inhibits the ability of SPPI to increase CD29 $\beta$ I integrin subunit expression on HIV-infected (GFP+) and bystander cells. TZM--bl cells were first transfected with the SPP/wt expression plasmid followed by overnight infection with HIVSFI62--R3 GFP.The next day the medium

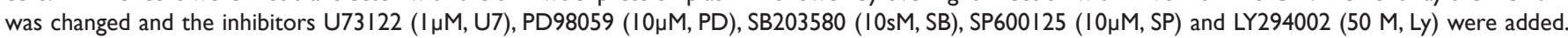
The cells were harvested 3 days later for flow cytometric analyses of CD29 expression. One--way ANOVA with significance of $p<.05$ was used. $* * * * p<.0001$, $* * * p<.0005, * * p<.005, * p<.05$. 
Signaling interactions between osteopontin and other human viruses have been reported, highlighting the potential importance of this early innate immune pathway in immunity. Interestingly, injury and inflammation of hepatocytes induced by hepatitis $\mathrm{C}$ virus results in elevated OPN expression in a mechanism involving the activation of MAP kinases including JNK, PI-3K, and p38. ${ }^{12}$ The Tax protein of human T-cell leukemia virus type 1 activates the OPN promoter through a PI-3K/AKT-dependent pathway. ${ }^{13}$ Moreover, the authors went on to link Tax signaling to an OPN/ CD44 receptor pathway that induces NF- $\mathrm{KB}$ activation. ${ }^{14}$

This report describes to our knowledge, the first systematic analyses of OPN motifs using a molecular approach combined with functional studies. OPN is an abundant secreted protein and its propensity to be present in biological fluids in truncated forms lead to early studies to determine whether these fragments possessed functional activity. ${ }^{5,8,15}$ Indeed, cleavage of OPN by thrombin results in two proteins that can modulate the balance of IL-12 and IL-10 signaling, thus influencing T-cell mediated immunity. ${ }^{8}$ With this background and building upon our finding that in macrophages OPN significantly enhances HIV replication, we sought to map in OPN the determinants of this function. However, our results suggest that the phenotype is multi factorial involving non-contiguous domains of OPN and likely at least two different signaling pathways. Interestingly, our results suggest the possibility that OPN takes advantage of integrin receptor signaling, which have the unique ability to function through insideout or outside-in signaling mechanisms. ${ }^{16,17}$ In this regard, the OPNa and $\mathrm{OPNb}$ (used in this study) isoforms were found to possess the capacity to activate integrin signaling while the OPNc variant did not. ${ }^{9}$ A recent study found that blocking of integrins including $\beta 1$, inhibited HIV infection of cervical mucosa immune cells. ${ }^{18}$ The N-terminal fragment of OPN was found to be better at binding to specific $\beta 1$ integrins in hetero dimeric complexes compared to the full-length protein. ${ }^{19}$ Moreover, the adhesion and integrin-binding abilities of OPN can be physically separated. ${ }^{19}$ As a secreted protein that can be found in the extracellular matrix, OPN function may also be regulated by matrix metalloproteinase (MMPs), for which cleavage sites have been identified. ${ }^{5}$ In this regard, a strong association between MMPs and HIV-associated dementia and brain atrophy has been shown. ${ }^{20-22}$

A limitation of the current study is the use of a surrogate cell line instead of primary macrophages. In this regard, the infectivity as measured by an increase in the number of GFP + cells is variable and modest, compared to the phenotype in macrophages treated with antiSPP1 siRNA, which is very reproducible. ${ }^{1}$ Some of the variability may be due to the high fusogenicity that is seen with TZM-bl cells and the tendency to underestimate the number of infected cells. Lastly, OPN is also regulated by post-translational modifications such as Nand O-linked glycosylation and phosphorylation, which could differ in a cell-type dependent manner.

\section{Conclusion}

Only relatively few founder viral particles actually successfully enter and infect the host at the mucosal barrier. ${ }^{23}$ Therefore, in order to overcome the bottleneck, HIV must harness multiple host pathways linking to cellular processes including cell survival under stress, proliferation, and migration in order to spread in a productive fashion. As such, HIV has evolved to use varied mechanisms of immune cells and the proinflammatory osteopontin pathway appears to be one of importance in helping to create a productive environment for HIV replication and spread through engaging integrin and MAPK signaling.

\section{Acknowledgement}

We would like to acknowledge Patrick Stoiber for his interest in this project and funding from the National Institutes of Mental Health (NIMH) MH095646 to AB.

\section{Conflict of interest}

Authors declare that there is no conflicts of interest.

\section{References}

1. Brown A, Islam T, Adams R, et al. Osteopontin enhances HIV replication and is increased in the brain and cerebrospinal fluid of HIV-infected individuals. J Neurovirol. 2011;17(4):382-392.

2. Roberts ES, Zandonatti MA, Watry DD, et al. Induction of pathogenic sets of genes in macrophages and neurons in NeuroAIDS. Am J Pathol. 2003;162(6):2041-2057.

3. Burdo TH, Ellis RJ, Fox HS. Osteopontin is increased in HIV-associated dementia. J Infect Dis. 2008;198(5):715-722.

4. Burdo TH, Wood MR, Fox HS. Osteopontin prevents monocyte recirculation and apoptosis. J Leukoc Biol. 2007;81(6):1504-1511.

5. Wang KX, Denhardt DT. Osteopontin: Role in immune regulation and stress responses. Cytokine Growth Factor Rev. 2008;19(5-6):333-345.

6. Brown A. Osteopontin: A key link between immunity, inflammation and the central nervous system. Transl Neurosci. 2012;3(3):288-293.

7. Wai PY, Kuo PC. The role of osteopontin in tumor metastasis. J Surg Res. 2004;121(2):228-241.

8. Ashkar S, Weber GF, Panoutsakopoulou V, et al. Eta-1 (Osteopontin): An early component of type-1 (cell-mediated) immunity. Science. 2000;287(5454): 860-864

9. Gimba ER, Tilli TM. Human osteopontin splicing isoforms: known roles, potential clinical applications and activated signaling pathways. Cancer Lett. 2013;31(1):11-17.

10. Shinohara ML, Kim HJ, Kim JH, et al. Alternative translation of osteopontin generates intracellular and secreted isoforms that mediate distinct biological activities in dendritic cells. Proc Natl Acad Sci U.S.A. 2008;105(20):7235-7239.

11. Brown A, Gartner S, Kawano T, et al. HLA-A2 down-regulation on primary human macrophages infected with an M-tropic EGFP-tagged HIV-1 reporter virus. J Leukoc Biol. 2005;78(3):675-685.

12. Iqbal J, McRae S, Banaudha $\mathrm{K}$, et al. Mechanism of hepatitis $\mathrm{C}$ virus (HCV)-induced osteopontin and its role in epithelial to mesenchymal transition of hepatocytes. J Biol Chem. 2013;288(52):36994-37009.

13. Zhang J, Yamada O, Matsushita Y, et al. Transactivation of human osteopontin promoter by human T-cell leukemia virus type 1-encoded Tax protein. Leuk Res. 2010;34(6):763-768.

14. Zhang J, Yamada O, Kida S, et al. Identification of CD44 as a downstream target of noncanonical NF- $\mathrm{kB}$ pathway activated by human T-cell leukemia virus type 1-encoded Tax protein. Virology. 2011;413(2):244-252.

15. Denhardt DT, Guo X. Osteopontin: A protein with diverse functions. FASEB J. 1993;7(15):1475-1482. 
16. Barczyk M, Carracedo S, Gullberg D. Integrins. Cell Tissue Res. 2010;339(1):269-280

17. Kim C, Ye F, Ginsberg MH. Regulation of integrin activation. Annu Rev Cell Dev Biol. 2011;27:321-345.

18. Tjomsland V, Ellegard R, Kjolhede P, et al. Blocking of integrins inhibits HIV-1 infection of human cervical mucosa immune cells with free and complement-opsonized virions. Eur J Immunol. 2013;43(9):2361-2372.

19. Yokosaki Y, Tanaka K, Higashikawa F, et al. Distinct structural equirements for binding of the integrins alphavbeta6, alphavbeta3, alphavbeta5, alpha5beta1 and alpha9beta1 to osteopontin. Matrix Biol. 2005;24(6):418427.
20. Ragin AB, Wu Y, Ochs R, et al. Serum matrix metalloproteinase levels correlate with brain injury in human immunodeficiency virus infection. $J$ Neurovirol. 2009; 15(3):275-281.

21. Ragin $\mathrm{AB}, \mathrm{Wu} \mathrm{Y}$, Ochs $\mathrm{R}$, et al. Marked relationship between matrix metalloproteinase 7 and brain atrophy in HIV infection. $J$ Neurovirol. 2011;17(2):153-158.

22. Conant K, McArthur JC, Griffin DE, et al. Cerebrospinal fluid levels of MMP-2, 7, and 9 are elevated in association with human immunodeficiency virus dementia. Ann Neuro. 19991 ;46(3):391-398.

23. Fraser C, Lythgoe K, Leventhal GE, et al. Virulence and pathogenesis of HIV-1 infection: an evolutionary perspective. Science 2014;343(6177):1243727 